\title{
Quality improvement project for managing elevated blood pressure in a primary care setting
}

This article was published in the following Dove Press journal: Integrated Blood Pressure Control

13 October 2017

Number of times this article has been viewed

\section{Stephanie M Thomas' Holly B Cassells ${ }^{2}$}

'Vital Life Wellness Center, ${ }^{2}$ lla Faye School of Nursing, University of the Incarnate Word, San Antonio, TX, USA

Video abstract

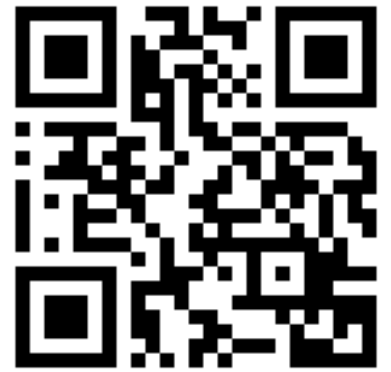

Point your SmartPhone at the code above. If you have a QR code reader the video abstract will appear. Or use: http://youtu.be/ciSDillGEbw
Correspondence: Holly B Cassells lla Faye School of Nursing, University of the Incarnate Word, 430I Broadway, NB 139, San Antonio, TX 78209, USA Tel + | 210-829-3977

Fax +| 210-829-3174

Email: cassells@uiwtx.edu
Abstract: Elevated blood pressure (BP) and prehypertension increase the risk of cardiovascular diseases, a national health concern. This article presents a quality improvement project implemented within a primary care setting that aimed at lowering cardiovascular risk by improving the identification, treatment, and follow-up of patients with elevated BP. This project was designed and implemented to address the identified deficiencies contributing to poor identification and follow-up of patients with elevated BP. The intervention was multi-pronged and comprised a staff educational program, introduction of a new method for measuring BP using the BpTRUTM device, and patient educational intervention. A significant improvement in staff BP knowledge scores was achieved following the intervention $(p<0.05)$. Patient participants also exhibited a significant improvement in post-intervention BP measurements $(p<0.05)$. This project showed that the implementation of a quality improvement project in a primary care setting can lead to significant improvements in staff BP knowledge and patient BP readings. However, future research in this area is required to determine whether particular lifestyle changes are directly associated with the reduction in BP.

Keywords: prehypertension, elevated blood pressure, BpTRU ${ }^{\mathrm{TM}}$, quality improvement

\section{Introduction}

Elevated blood pressure (BP) is a risk factor for cardiovascular disease (CVD). ${ }^{1}$ CVD is a leading cause of death in the United States. ${ }^{2}$ Guidelines aimed at reducing CVD prevalence that target individuals with prehypertension have been in effect for years. Healthy People 2020 aims to improve cardiovascular health, ${ }^{3}$ including a $2 \%$ increase in the number of adults who have had their BP measured in the last 2 years and who understand whether the reading is normal or high. ${ }^{3}$ Its second aim is to increase the number of individuals with prehypertension who meet recommendations for body mass index, physical activity, and saturated fat, alcohol, and sodium consumption. ${ }^{3}$

The Seventh Report of the Joint National Committee on Prevention, Detection, Evaluation, and Treatment of High Blood Pressure (JNC 7) guidelines discuss proper BP measurement technique, define prehypertension as a systolic pressure of 120-139 $\mathrm{mmHg}$ and/or a diastolic pressure of $80-89 \mathrm{mmHg}$, and recommend lifestyle modifications and periodic repeat screening for patients with BP readings within or above this range. ${ }^{4}$ Recently, JNC 8 guidelines were released with a shift in focus toward hypertension treatment. Unlike in JNC 7 guidelines, guidance pertaining to BP measurement, prehypertension or hypertension definitions, and interventions recommended for 
prehypertension are absent..$^{5}$ Nevertheless, many recent studies continue to support prehypertension as a CVD riskincreasing factor. Notably, a recent meta-analysis pooled data from 18 prospective cohort studies involving over 460,000 subjects and found that prehypertension is associated with a high CVD risk. ${ }^{6}$ Furthermore, prehypertension prevalence in adults is $\sim 36 \%{ }^{7}$ The significant CVD and prehypertension prevalence presents an important opportunity to develop cardiovascular risk-reducing interventions. Unfortunately, health care systems continue to struggle with reducing disease risk despite awareness regarding these issues and access to nationally published and recognized guidelines on heart disease risk.

Comprehensive assessment of a primary care clinic in a large southern metropolitan area revealed many opportunities for interventions aimed at addressing prehypertension more effectively. Although nearly all patients had a BP recorded at every visit, $\sim 30 \%$ patients had one or more elevated BP readings, meeting the prehypertension or hypertension criteria, but were not diagnosed or managed. Approximately $50 \%$ of patients with an elevated BP reading did not receive appropriate intervention, such as lifestyle modification advice, recommendations for home BP monitoring, or follow-up instructions. In addition, various deviations from the proper BP measurement technique, as established by the American Heart Association Guidelines, including using uncalibrated manual sphygmomanometers, were observed. ${ }^{8}$ Discrepancies in measurement technique are concerning because routine, in-office, manual BP measurements without careful adherence to proper technique can yield considerably inaccurate results. ${ }^{9,10}$ Manual BP assessments also fail to differentiate normotensive patients from those with white-coat or masked hypertension, ${ }^{10}$ further complicating the problem of accurately identifying patients at risk. Other opportunities for improvement were identified when the staff and patients' knowledge about BP was assessed. Patient surveys revealed that $76 \%$ demonstrated lack of knowledge. Medical assistant staff who typically performed BP screening also completed a BP quiz wherein most questions answered erroneously concerned proper BP measurement technique.

\section{Quality improvement project}

The primary care setting, focused on in this study, is located in an urban area of San Antonio, Texas. Although this is a family practice setting, the clinic mission is to promote wellness and preventive care, with strategic plans to move from a disease-focused model toward a preventive care model to reduce disease risk and improve health outcomes. The majority of over 5,000 patients in the clinic have private insurance (81\%), with self-pay or uninsured accounting for $11 \%$ and Medicare for $8 \%$. Most patients are adult females (78\%) aged 18-64 years (86\%). Before the intervention, 9\% of patients were diagnosed with prehypertension, whereas chart reviews suggested that an additional 30\% met prehypertension or hypertension criteria but had no diagnosis indicated in the medical record. This was the primary rationale for developing a quality improvement project to manage elevated BP in this setting.

An important first step for reducing cardiovascular risk is accurately identifying patients with elevated BP, allowing prescription of interventions for reducing BP. Given the discrepancies noted in the clinic's routine measurement technique and further assessment of staff and patients' concerns revealed during the initial assessment of the study site, a more accurate and time-efficient method for assessing BP is warranted.

Strong evidence was found in several studies on 1,730 patients supporting the accuracy of the in-office automated oscillometric BP measurement device BpTRUTM (BpTRU Medical Devices, Coquitlam, Canada). ${ }^{9-13}$ BpTRU ${ }^{\text {TM }}$ reduced measurement errors caused by improper technique ${ }^{9}$ and the white-coat effect, which is the presence of elevated BP in otherwise normotensive individuals when measured in a medical office. ${ }^{10}$ It was determined that these two benefits of BpTRUTM would provide a justification for using an alternative to the manual measurement technique currently used at the study site. The BpTRUTM cuff is applied to the patient's arm in the usual fashion; six BP measurements, 1-minute apart, are recorded, discarding the first result and averaging the remaining five to provide a result accurate to $3 \mathrm{mmHg}$, as per manufacturer specifications. In addition, using this method instead of perfecting the current manual technique saves time, as BpTRUTM neither requires the patient to rest for 5 minutes before measurement nor extensive staff training, as required for the manual technique which necessitates several steps to ensure accuracy. ${ }^{12}$ This method, which is more accurate than the routine BP-measuring method, saves time by avoiding repeat measurements at the end of the visit, which has been the standard procedure in this setting. Therefore, BpTRUTM was selected by clinic providers as the method to be introduced to measure BP for the quality improvement project.

This project aimed to improve the identification and treatment of patients with elevated BP readings. To evaluate the success of this quality improvement project, four study objectives were developed: 
1. Increase the percentage of electronic medical records, with documentation of an elevated BP, from $9 \%$ to $20 \%$, as indicated by an appropriate ICD code for patients previously undiagnosed with hypertension.

2. Reduce the percentage of patients with elevated BP who did not receive proper intervention and follow-up from $50 \%$ to $25 \%$.

3. Demonstrate a statistically significant increase in staff's knowledge about BP following an educational intervention.

4. Demonstrate a statistically significant increase in patient knowledge about BP following the intervention.

\section{Methods}

This quality improvement project was developed by a nurse practitioner who was a Doctor of Nursing Practice graduate student. It was approved by the University of the Incarnate Word Institutional Review Board (14-05-007) and the study site administration. Following approval, a quasi-experimental study with two intervention components was conducted. Both the components utilized volunteer participants who were not randomized to intervention or treatment groups but served as their own controls.

The first component was the education and training of medical assistant clinic staff in the identification and management of patients with elevated BP. Each medical assistant employed at the study site was asked to participate in the training program because it included training on BpTRUTM as a job requirement at the site. Participation in the pre- and postintervention testing was completely voluntary, and staff was informed that declining to participate would not affect their employment status. Eleven medical assistant staff members chose to participate and provided written informed consent before pretest administration. Staff pre- and posttests were not identified by name but by a number drawn from a hat. A matched staff roster and the identifying number was kept in a locked file accessible only to the investigator and used only for project evaluation purposes. A staff educational program was held to acquaint staff participants with BpTRUTM and present them with general information about BP monitoring and risks associated with elevated BP. The $\sim 1$-hour-long educational program comprised group discussion, lecture, and hands-on training of BpTRUTM. At the beginning of the session, the medical assistants completed a written pretest, including demographic questions. The lesson plan for staff education was adapted from "Measure up, pressure down: Provider toolkit to improve hypertension control," an evidence-based campaign developed with the support of several national organizations to help health systems achieve hypertensive control in $80 \%$ of patients by $2016 .{ }^{14}$ The lesson plan was adapted to include BpTRU ${ }^{\mathrm{TM}}$ using the manufacturer's instructions. The staff was also acquainted with the purpose and protocol of the elevated BP project. Two weeks later, a follow-up session was held with the medical assistant staff to discuss their perceptions on the project, to identify and remedy any concerns regarding the intervention, and to administer the posttest to assess learning.

The second component utilized a convenience sample of patient volunteers who visited the clinic for routine care during a 2-month time frame and met the criteria of having a BpTRUTM average BP of $\geq 120 / 80 \mathrm{mmHg}$, no diagnosis of hypertension, and no current treatment for hypertension. Eligible patients were identified and invited to participate by the clinic staff. Forty-five eligible patients agreed to participate and provided written consent. A power-based sample-size calculation indicated that a sample size of 34 was required to demonstrate a medium effect size. Therefore, all 45 eligible patient volunteers were enrolled, of which nine did not follow-up, leaving a final sample size of 36 who completed the study. Patients were initially assessed for eligibility by the medical assistant who obtained a BpTRUTM measurement at the beginning of the visit. The providers were instructed to review the average BpTRUTM reading and record the appropriate ICD-9 code for BPs of $\geq 120 / 80 \mathrm{mmHg}$ in the electronic medical record. Patients with elevated BP without a diagnosis of hypertension who were not on any antihypertensive medication were asked to participate. Those who agreed attempted a BP awareness pretest, including a demographic and lifestyle survey to assess tobacco use, alcohol use and frequency, and exercise frequency, developed by the principal investigator. At the end of the visit, patients were provided an educational handout, which was adapted from "Measure up, pressure down: provider toolkit to improve hypertension control" from the American Medical Group Foundation. ${ }^{14}$ It explained BP classifications, elevated BP risks, and lifestyle modifications to help reduce BP. This educational information is consistent with the general consensus of the American Heart Association, JNC 7 guidelines and current treatment recommendations for patients with elevated BP. Patients with an elevated BP diagnosis were also provided an option to participate in an existing lifestyle modification program offered by the clinic, which teaches heart-healthy diet and menu planning consistent with all current lifestyle recommendations of the American Heart Association. ${ }^{15}$ Patients were then asked to return in 6-12 weeks for BP re-assessment using BpTRUTM and to repeat the BP awareness posttest to complete the study. 
Descriptive statistics, including means and percentages, were used to describe sample demographic characteristics, categorical measures, knowledge scores, and BPs. $T$ tests were applied to pre- and post-intervention data to determine statistically significant improvements in BP. All data were analyzed using the SPSS v.22 software.

\section{Results}

Of the 11 staff participants, 10 were females $(90.9 \%)$ and 1 was male (Table 1). The participants' ages were 22-37 years (mean, 29 years; $\mathrm{SD}=4.76$ ), and their work experience ranged from 6 months to 13 years (mean, 6.227 years; SD $=4.77$ ). Of the patient participants, no statistically significant differences in age, gender, race, ethnicity, or lifestyle factors were observed between participants who did $(n=36)$ and did not $(\mathrm{n}=9)$ complete the study (Table 2). Participants, with a mean age of $49.25(\mathrm{SD}=11.7)$ years, were primarily females (75\%) and non-Hispanic (58.3\%).

This quality improvement project demonstrated that intervening to increase the identification and treatment of patients with elevated BP yields statistically significant improvements in systolic BP (Figure 1) and diastolic BP (Figure 2) postintervention. The mean pre-intervention systolic and diastolic BPs were $132(\mathrm{SD}=11.7)$ and $85(\mathrm{SD}=6.6) \mathrm{mmHg}$, respectively. The mean post-intervention systolic and diastolic BPs had reduced to $124(\mathrm{SD}=12.1)$ and $81(\mathrm{SD}=8.5) \mathrm{mmHg}$, respectively (paired $t$-tests: systolic pressure, $t[31]=3.096$, $p=0.004$; diastolic pressure, $t[31]=3.625, p=0.001$ ) (Table 3 ). Charts were reviewed to determine the percentage of patients with an elevated BP reading post-intervention who did not have an appropriate intervention or follow-up instructions documented in the visit plan. This number was compared with the pre-intervention value of $50 \%$. Post-intervention, this value decreased to $17 \%$, indicating that objective 2 was met.

In addition, objective 3 , which sought to increase staff's BP knowledge, was met. An analysis of staff's knowledge before and after the nurse practitioner-led educational session indicated a statistically significant increase in mean knowledge scores from $73 \%$ at baseline to $85 \%$ post-intervention $(t[10]=2.371, p=0.039)$ (Table 1). Additional analyses were conducted to determine associations between knowledge and demographic characteristics using Pearson's correlations. No significant correlations of age, gender, or years of experience with test scores were revealed (data not shown).

Objective 4, which aimed to increase patients' knowledge about BP, was not met post-intervention. Of the three knowledge assessment questions, the mean pre-intervention knowledge score was 2.25 ( $\mathrm{SD}=0.692$ ) compared with a slightly lower post-intervention mean knowledge score of $2.139(\mathrm{SD}=0.683)$, although this decline was not statistically significant.

Lastly, objective 1, which aimed to increase the percentage of electronic medical records in the clinic with documentation of an elevated BP from a baseline of $9 \%$ to $20 \%$, was not met because post-intervention review revealed that

Table 2 Patient characteristics

\begin{tabular}{|c|c|c|}
\hline Measure & Completed & Dropout \\
\hline \multicolumn{3}{|l|}{ Gender, n (\%) } \\
\hline Male & $9(25.0 \%)$ & $3(33.3 \%)$ \\
\hline Female & 27 (75.0\%) & $6(66.7 \%)$ \\
\hline \multicolumn{3}{|l|}{ Ethnicity, n (\%) } \\
\hline Not Hispanic/Latino & $21(58.3 \%)$ & $4(44.4 \%)$ \\
\hline Hispanic/Latino & $15(41.7 \%)$ & $5(55.6 \%)$ \\
\hline \multicolumn{3}{|l|}{ Race, n (\%) } \\
\hline American Indian/Alaskan Native & $2(5.6 \%)$ & \\
\hline Asian & I (2.8\%) & \\
\hline White & $33(91.7 \%)$ & $9(100.0 \%)$ \\
\hline \multicolumn{3}{|c|}{ Exercise frequency (times per week), n (\%) } \\
\hline 0 times & $9(25.0 \%)$ & I (II.I\%) \\
\hline I-2 times & $21(58.3 \%)$ & $6(66.7 \%)$ \\
\hline $3-4$ times & $3(8.3 \%)$ & I (II.I\%) \\
\hline $5+$ times & $3(8.3 \%)$ & I (II.I\%) \\
\hline \multicolumn{3}{|l|}{ Tobacco, n (\%) } \\
\hline No & $33(91.7 \%)$ & $9(100.0 \%)$ \\
\hline Yes & $3(8.3 \%)$ & \\
\hline \multicolumn{3}{|l|}{ Alcohol, n (\%) } \\
\hline No & $16(44.4 \%)$ & I (II.I\%) \\
\hline Yes & $20(55.6 \%)$ & 8 (88.9\%) \\
\hline \multicolumn{3}{|l|}{ Drinks per week, n (\%) } \\
\hline 0 & $16(44.4 \%)$ & I (II.I\%) \\
\hline $\mathrm{I}-2$ & $13(36.1 \%)$ & $5(55.6 \%)$ \\
\hline $3-4$ & $3(8.3 \%)$ & $2(22.2 \%)$ \\
\hline $5-6$ & $3(8.3 \%)$ & \\
\hline $7+$ & I (2.8\%) & I (II.I\%) \\
\hline
\end{tabular}

Table I Staff characteristics and test scores

\begin{tabular}{|c|c|c|c|c|c|c|}
\hline Measure & Mean & Median & SD & Range & Minimum & Maximum \\
\hline Age (years) & 29.091 & 30.000 & 4.763 & 15.000 & 22.000 & 37.000 \\
\hline Experience (years) & 6.227 & 7.000 & 4.772 & 12.500 & 0.500 & 13.000 \\
\hline Pretest (\%) & 72.8 & 78.0 & 0.214 & 67.0 & 33.0 & 100.0 \\
\hline Posttest (\%) & 84.9 & 89.0 & 0.200 & 56.0 & 44.0 & 100.0 \\
\hline Pretest correct, n & 6.545 & 7.000 & 1.916 & 6.000 & 3.000 & 9.000 \\
\hline Posttest correct, n & 7.636 & 8.000 & $\mathrm{I} .804$ & 5.000 & 4.000 & 9.000 \\
\hline
\end{tabular}




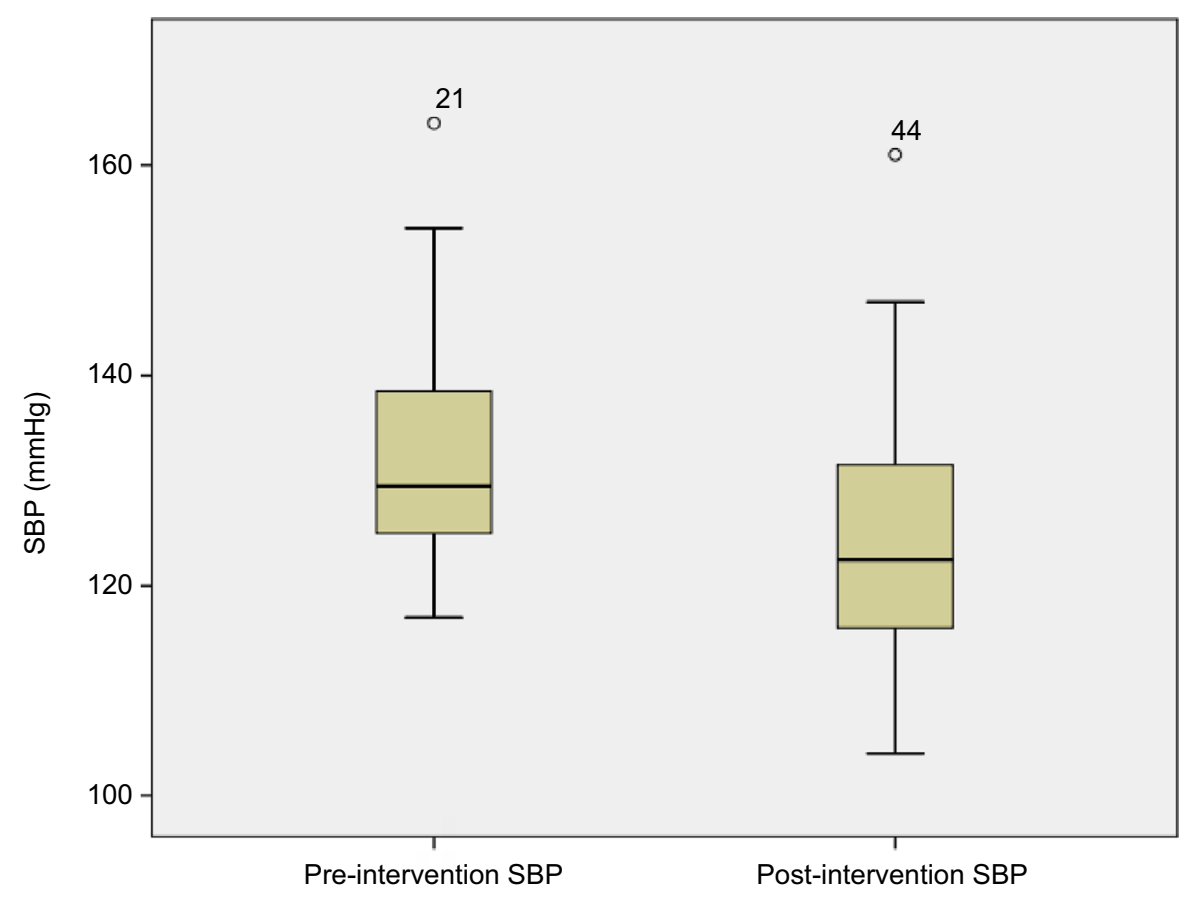

\begin{tabular}{|l|r|r|r|r|r|r|}
\hline \multicolumn{1}{|c|}{} & \multicolumn{9}{|c|}{ Case processing summary } \\
\cline { 2 - 8 } & \multicolumn{2}{|c|}{ Valid } & \multicolumn{2}{c|}{ Cases } \\
\cline { 2 - 8 } & $\mathrm{N}$ & $\%$ & $\mathrm{~N}$ & $\%$ & $\mathrm{~N}$ & \multicolumn{2}{c|}{ Total } \\
\hline preSBP & 32 & 71.1 & 13 & 28.9 & 45 & 100.0 \\
postSBP & 32 & 71.1 & 13 & 28.9 & 45 & 100.0 \\
\hline
\end{tabular}

Figure I SBP levels.

Abbreviation: SBP, systolic blood pressure.

only $13 \%$ of patients with elevated BP had the appropriate ICD code entered in the medical record.

Subsequent analyses were conducted to determine whether any significant correlations existed among knowledge scores, BP readings, lifestyle factors, or demographics, as well as differences among patients who participated in the lifestyle-modification program. One statistically significant finding was related to exercise frequency, with a pre-intervention mean of $1.000(\mathrm{SD}=0.828)$ and a posttest mean of 1.389 ( $\mathrm{SD}=0.871$ ), suggesting an increase in exercise frequency post-intervention $(t[35]=2.348, p=0.025)$; however, this frequency did not correlate with BP readings. In addition, a significantly higher mean knowledge score $(t[32]=2.179, p=0.037)$ was found in patients who did not use tobacco $(2.273, \mathrm{SD}=0.719)$ than in those who did (2.000, $\mathrm{SD}=0.000)$. No significant differences were found in knowledge scores or BP readings between the 13 patients who attended the lifestyle-modification program and those who did not. No other statistically significant findings related to demographics or lifestyle factors were found.

\section{Discussion}

In addition to the marked improvement in identifying patients previously undiagnosed with prehypertension, a notable success of the project was the statistically significant improvement in post-intervention BP readings from pre-intervention readings. Although specific lifestyle factors, namely exercise, smoking, and alcohol, were not individually correlated with reductions in BP, each patient received a comprehensive introduction to elevated BP and its associated risks. We believe that lifestyle changes may have occurred because of this intervention, especially considering the statistically significant increase in exercise frequency among patient participants post-intervention. In addition, the intervention may have contributed to the adoption of other lifestyle changes not assessed in this study, which has the propensity to improve 


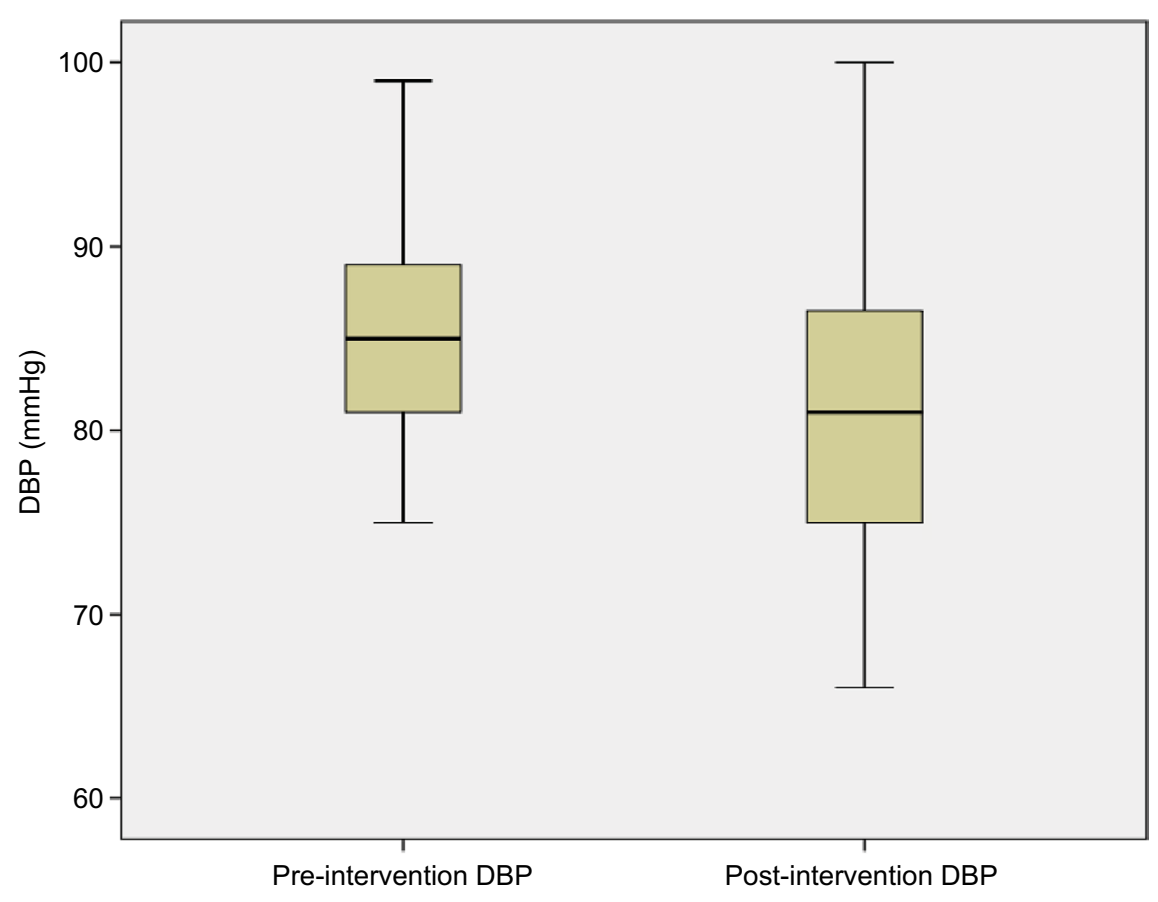

\begin{tabular}{|l|r|r|r|r|r|r|}
\hline \multicolumn{1}{|c|}{ Case processing summary } \\
\cline { 2 - 8 } & \multicolumn{2}{|c|}{ Valid } & \multicolumn{2}{c|}{ Missing } & \multicolumn{2}{c|}{ Total } \\
\cline { 2 - 8 } & \multicolumn{1}{|c|}{$\mathrm{N}$} & $\%$ & $\mathrm{~N}$ & $\%$ & $\mathrm{~N}$ & $\%$ \\
\hline preDBP & 32 & 71.1 & 13 & 28.9 & 45 & 100.0 \\
postDBP & 32 & 71.1 & 13 & 28.9 & 45 & 100.0 \\
\hline
\end{tabular}

Figure 2 DBP levels.

Abbreviation: DBP, diastolic blood pressure.

BP. For example, sodium restriction and weight loss were not assessed. Surprisingly, data analysis revealed that patient BP knowledge scores did not improve post-intervention. These findings are consistent with those of Lauzière et al, ${ }^{16}$ wherein a patient educational program was associated with improved systolic BPs but not improved patient knowledge.

Improving medical assistants' knowledge and skills is crucial as they perform most initial screening activities. This study demonstrated that even when baseline hypertension knowledge is limited among staff, these levels can be increased through simple yet effective educational training methods. Posttest knowledge scores significantly improved by an average of 12 points over pretest scores, substantiating the use of staff educational programs in the clinical setting.

\section{Limitations}

Several challenges were identified in the development and implementation of this quality improvement project to manage elevated BP. First, difficulties were initially encountered in achieving complete staff and provider participation for identifying and scheduling follow-up visits for patients with elevated BPs. Therefore, a focused effort at changing the clinic's standard of care and promoting the intervention was needed. Frequent staff and provider engagement was required to implement and sustain the project. Fortunately, with time, the staff and providers valued the use of BpTRUTM for its accuracy, ease of use, and assistance in making clinical decisions, and the entire intervention started to become second nature.

Another challenge during the project was the need to increase the patient-friendliness of the patient survey tool. Some questions could have been phrased differently to enhance patient understanding. Issues regarding question complexity were not identified until the final data-collection phase when some surveys were completed via telephone and participants often asked the surveyor for more information or clarification.

Finally, the generalizability of this study is limited to patients in a primary care setting that is focused on wellness. Patients who seek care in such a setting may have differences in motivation, health knowledge, and resources compared with those in an average primary care setting. Furthermore, 
Table 3 Pre- and post-intervention blood pressure readings

\begin{tabular}{|c|c|c|c|c|c|c|}
\hline & PreSBP (mmHg) & PreDBP $(\mathrm{mmHg})$ & PostSBP* (mmHg) & PostDBP $(\mathrm{mmHg})$ & Age (years) & Gender \\
\hline $\mathrm{I}$ & 154 & 99 & 133 & 88 & 46 & $\mathrm{~F}$ \\
\hline 2 & 122 & 83 & 114 & 78 & 53 & $M$ \\
\hline 3 & 130 & 85 & 136 & 95 & 38 & $\mathrm{~F}$ \\
\hline 4 & 125 & 88 & & & 55 & $M$ \\
\hline 5 & 129 & 86 & 130 & 86 & 60 & $M$ \\
\hline 6 & 123 & 80 & & & 37 & $\mathrm{~F}$ \\
\hline 7 & 122 & 75 & 124 & 86 & 22 & $M$ \\
\hline 8 & 121 & 83 & 112 & 83 & 49 & $\mathrm{~F}$ \\
\hline 9 & 139 & 94 & 116 & 84 & 54 & $\mathrm{~F}$ \\
\hline 10 & 160 & 85 & & & 45 & $\mathrm{~F}$ \\
\hline II & $13 \mid$ & 90 & 132 & 88 & 39 & $\mathrm{~F}$ \\
\hline 12 & 127 & 81 & 119 & 75 & 51 & $\mathrm{~F}$ \\
\hline 13 & 126 & 76 & 116 & 71 & 61 & $\mathrm{~F}$ \\
\hline 14 & 112 & 82 & & & 32 & $M$ \\
\hline 15 & 140 & 93 & 127 & 83 & 41 & $\mathrm{~F}$ \\
\hline 16 & 126 & 88 & 115 & 81 & 32 & $M$ \\
\hline 17 & 135 & 85 & 121 & 77 & 59 & $\mathrm{~F}$ \\
\hline 18 & $|3|$ & 86 & 130 & 78 & 36 & $F$ \\
\hline 19 & 122 & 79 & & & 48 & $F$ \\
\hline 20 & $14 \mid$ & 98 & 128 & 87 & 53 & $M$ \\
\hline 21 & 164 & 94 & $13 \mid$ & 80 & 59 & $\mathrm{~F}$ \\
\hline 22 & 127 & 86 & & & 53 & $F$ \\
\hline 23 & 135 & 91 & & & 21 & $M$ \\
\hline 24 & 117 & 83 & 109 & 75 & 49 & $\mathrm{~F}$ \\
\hline 25 & 140 & 83 & & & 59 & $F$ \\
\hline 26 & 114 & 80 & & & 60 & $M$ \\
\hline 27 & 120 & 81 & 112 & 77 & 31 & $\mathrm{~F}$ \\
\hline 28 & 125 & 79 & 123 & 72 & 68 & $M$ \\
\hline 29 & 147 & 80 & 104 & 66 & 59 & $\mathrm{~F}$ \\
\hline 30 & $13 \mid$ & 81 & 147 & 73 & 65 & $F$ \\
\hline 31 & 125 & 78 & 116 & 70 & 58 & $M$ \\
\hline 32 & 126 & 85 & & & $4 I$ & $\mathrm{~F}$ \\
\hline 33 & $13 \mid$ & 75 & 117 & 68 & 49 & $F$ \\
\hline 34 & 146 & 85 & 114 & 76 & 67 & $F$ \\
\hline 35 & 126 & 85 & 142 & 100 & 33 & $M$ \\
\hline 36 & 137 & 86 & $13 \mid$ & 84 & 60 & $\mathrm{~F}$ \\
\hline 37 & 121 & 88 & 118 & 81 & 42 & $F$ \\
\hline 38 & 129 & 82 & 117 & 70 & 47 & $F$ \\
\hline 39 & 123 & 84 & 122 & 84 & 39 & $F$ \\
\hline 40 & 138 & 98 & 134 & 92 & 32 & $F$ \\
\hline $4 I$ & 145 & 103 & & & 36 & $F$ \\
\hline 42 & 128 & 78 & & & 59 & $F$ \\
\hline 43 & 127 & 95 & 140 & 97 & 39 & $F$ \\
\hline 44 & 147 & 85 & 161 & 91 & 62 & $F$ \\
\hline 45 & 123 & 75 & & & 56 & $F$ \\
\hline
\end{tabular}

Notes: *No data have been entered for the post-intervention blood pressure readings of participants who did not present for follow-up and therefore were not included in the statistical analysis for this measure.

Abbreviations: SBP, systolic blood pressure; DBP, diastolic blood pressure; F, female; $M$, male.

the sample characteristics limit generalizability as the patient participants were primarily females and non-Hispanic.

\section{Implications}

Further research can be conducted in the area of quality improvement for identifying patients with an elevated BP and intervening, particularly focusing on the effects of lifestyle modification on BP readings. Utilizing a particular treatment regimen or developing a survey tool that assesses a wide range of lifestyle changes to manage elevated BPs may reveal strong associations with decreases in BP readings post-intervention. 
Patient follow-up is another area where future research in quality improvement is needed. In this project, getting many of the patients to return for their follow-up BP measurement was difficult. Although follow-up for elevated BP may not be a priority for many patients or even for many primary care clinics, it is nevertheless an important component of highquality, effective care.

\section{Conclusion}

This project was designed as a quality improvement initiative to manage elevated BP, affecting a large percentage of patients at urban primary care clinics. National efforts have been made to raise awareness and promote cardiovascular risk reduction; however, more efforts are required at the health care system level to mitigate this risk. The implementation of quality improvement projects incorporating current evidence can not only address unmet patient needs but also contribute to achieving national CVD risk-reduction goals. More efforts can be made in health care systems to better identify patients and implement effective preventive interventions for individuals who are at risk for disease, as opposed to taking a reactive stance and focusing only on disease treatment.

\section{Acknowledgment}

The BpTRUTM device utilized in the project was purchased by the study site utilizing its own private funds and remained the property of the site following the conclusion of the project.

\section{Disclosure}

The authors report no conflicts of interest in this work.

\section{References}

1. Linden B. NICE guidance on primary hypertension. BJCN. 2011;6(12):582-584.

2. Keenan N, Shaw K. Coronary heart disease and stroke deaths - United States, 2006. MMWR Surveill Summ. 2011;60(Suppl):62-66.
3. U.S. Department of Health and Human Services. Healthy People 2020. Washington, DC; 2010. Available from: http://www.healthypeople.gov. Accessed April 1, 2014.

4. Chobanian AV, Bakris GL, Black HR, et al. The seventh report of the joint national committee on prevention, detection, evaluation, and treatment of high blood pressure: the JNC 7 report. JAMA. 2003;289(19):2560-2571.

5. James PA, Oparil S, Carter BL et al. 2014 evidence-based guideline for the management of high blood pressure in adults report from the panel members appointed to the Eighth Joint National Committee (JNC 8). JAMA. 2014;311(5):507-520.

6. Huang Y, Wang S, Cai X, et al. Prehypertension and incidence of CVD: a meta-analysis. BMC Medicine. 2013;11(1):177.

7. Guo X, Zou L, Zhang X, et al. Prehypertension: a meta-analysis of the epidemiology, risk factors, and predictors of progression. Tex Heart Inst J. 2011;38(6):643.

8. Pickering TG, Hall JE, Appel LJ, et al. Recommendations for blood pressure measurement in humans and experimental animals part 1 : Blood pressure measurement in humans: a statement for professionals from the Subcommittee of Professional and Public Education of the American Heart Association Council on High Blood Pressure Research. Circulation. 2005;111(5):697-716.

9. Godwin M, Birtwhistle R, Delva D, et al. Manual and automated office measurements in relation to awake ambulatory blood pressure monitoring. Fam Pract. 2011;28(1):110-117.

10. Myers MG, Godwin M, Dawes M, et al. Conventional versus automated measurement of blood pressure in primary care patients with systolic hypertension: randomised parallel design controlled trial. $B M J$. 2011;342:1-9.

11. Brothwell S, Dutton M, Ferro C, Stringer S, Cockwell P. Optimising the accuracy of blood pressure monitoring in chronic kidney disease: the utility of BpTRU. BMC Nephrol. 2013;14(1):218-229.

12. Edwards C, Hiremath S, Gupta A, McCormick BB, Ruzicka M. BpTRUth: do automated blood pressure monitors outperform mercury? J Am Soc Hypertens. 2013;7(6):448-453.

13. Lamarre-Cliché M, Cheong NN, Larochelle P. Comparative assessment of four blood pressure measurement methods in hypertensives. Can J Cardiol. 2011;27(4):455-460.

14. American Medical Group Foundation. Measure up, pressure down: provider toolkit to improve hypertension control; 2013. Available from: http://www.measureuppressuredown.com/HCProf/Find/provToolkit_find.asp. Accessed October 12, 2013.

15. American Heart Association. Understanding blood pressure readings; 2012. Available from: http://www.heart.org/HEARTORG/Conditions/ HighBloodPressure/AboutHighBloodPressure/Understanding-BloodPressure-Readings_UCM_301764_Article.jsp. Accessed October 9, 2013.

16. Lauzière TA, Chevarie N, Poirier M, Utzschneider A, Bélanger M. Effects of an interdisciplinary education program on hypertension: a pilot study. Can J Cardiovasc Nurs. 2012;23(2):12-19.
Integrated Blood Pressure Control

\section{Publish your work in this journal}

Integrated Blood Pressure Control is an international, peer-reviewed open-access journal focusing on the integrated approach to managing hypertension and risk reduction. Treating the patient and comorbidities together with diet and lifestyle modification and optimizing healthcare resources through a multidisciplinary team approach constitute key

\section{Dovepress}

features of the journal. This journal is indexed on American Chemical Society's Chemical Abstracts Service (CAS). The manuscript management system is completely online and includes a very quick and fair peerreview system, which is all easy to use. Visit http://www.dovepress.com/ testimonials.php to read real quotes from published authors. 